\title{
BMJ Open Anxiety and depressive symptoms are associated with poor sleep health during a period of COVID-19-induced nationwide lockdown: a cross-sectional analysis of adults in Jordan
}

\author{
Yazan A Al-Ajlouni (D) ," Su Hyun Park (1) ," Jude Alawa (i) , ${ }^{3}$ Ghaith Shamaileh, ${ }^{4}$ \\ Aziz Bawab, ${ }^{5}$ Wafaa M El-Sadr, ${ }^{2,6}$ Dustin T Duncan ${ }^{2}$
}

To cite: Al-Ajlouni YA, Park SH, Alawa J, et al. Anxiety and depressive symptoms are associated with poor sleep health during a period of COVID-19-induced nationwide lockdown: a cross-sectional analysis of adults in Jordan. BMJ Open 2020;10:e041995. doi:10.1136/ bmjopen-2020-041995

- Prepublication history for this paper is available online To view these files, please visit the journal online (http://dx.doi. org/10.1136/bmjopen-2020041995).

Received 25 June 2020 Revised 28 September 2020 Accepted 12 November 2020

Check for updates

(C) Author(s) (or their employer(s)) 2020. Re-use permitted under CC BY-NC. No commercial re-use. See rights and permissions. Published by BMJ.

For numbered affiliations see end of article.

Correspondence to

Yazan A Al-Ajlouni;

yal_ajlo@student.nymc.edu

\section{ABSTRACT}

Background Jordan, a Middle Eastern country, declared a state of national emergency due to COVID-19 and a strict nationwide lockdown on 17 March 2020, banning all travel and movement around the country, potentially impacting mental health. This study sought to investigate the association between mental health (eg, anxiety and depressive symptoms) and sleep health among a sample of Jordanians living through a state of COVID-19-induced nationwide lockdown.

Methods Using Facebook, participants $(n=1240)$ in Jordan in March 2020 were recruited and direct to a web-based survey measuring anxiety (items from General Anxiety Disorder 7-item (GAD-7) scale instrument), depressive symptoms (items from Center for Epidemiologic Studies Depression Scale), sleep health (items from the Pittsburgh Sleep Quality Index) and sociodemographic. A modified Poisson regression model with robust error variance. Adjusted prevalence ratios (aPRs) and 95\% Cls were estimated to examine how anxiety and depressive symptoms may affect different dimensions of sleep health: (1) poor sleep quality, (2) short sleep duration, (3) encountering sleep problems.

Results The majority of participants reported having experienced mild $(33.8 \%)$, moderate $(12.9 \%)$ or severe $(6.3 \%)$ levels of anxiety during lockdown, and nearly half of respondents reported depressive symptoms during lockdown. Similarly, over $60 \%$ of participants reported having experienced at least one sleep problem in the last week, and nearly half reported having had short sleep duration. Importantly, anxiety was associated with poor sleep health outcomes. For example, corresponding to the dose-response relationship between anxiety and sleep health outcomes, those reporting severe anxiety were the most likely to experience poor sleep quality $(\mathrm{aPR}=8.95$; $95 \% \mathrm{Cl}=6.12$ to 13.08$)$, short sleep duration ( $\mathrm{aPR}=2.23$; $95 \% \mathrm{Cl}=1.91$ to 2.61 ) and at least one problem sleep problem (aPR=1.73; $95 \% \mathrm{Cl}=1.54$ to 1.95). Moreover, depressive symptoms were also associated with poor sleep health outcomes. As compared with scoring in the first quartile, scoring fourth quartile was associated with poor sleep quality (aPR=11.82; $95 \% \mathrm{Cl}=6.64$ to 21.04$)$, short sleep duration (aPR=1.87; $95 \% \mathrm{Cl}=1.58$ to 2.22 ),

\section{Strengths and Limitations of this study}

- This study used a relatively large sample for a population that is heavily understudied in public health research, as well as an increased number of exposure and outcome measures compared with the existing research conducted in the region.

- To the best of our knowledge, this is the first study to examine this association among a Middle Eastern population and one of the first to investigate the impact of an extremely strict extended lockdown on health outcomes.

- The reliance of subjective measures for both the exposure and the outcome may have introduced bias.

- The generalisability of the results is an acknowledged concern given that participants were Jordanian using the social media platform, Facebook.

and experiencing at least one sleep problem (aPR=1.90; $95 \% \mathrm{Cl}=1.66$ to 2.18$)$.

Conclusions Increased levels of anxiety and depressive symptoms can negatively influence sleep health among a sample of Jordanian adults living in a state of COVID-19induced nationwide lockdown.

\section{INTRODUCTION}

On 31 December 2019, a pneumonia of unknown cause was first detected in Wuhan, the largest metropolitan area in China's Hubei province, and was reported to the WHO Country Office in China. A month later, COVID-19 outbreak was declared as a public health emergency of international concern by WHO. On 2 March 2020, Jordan detected its first COVID-19 case in the country. On 11 March 2020, as the number of COVID-19 cases outside China continued to increase, resulting in over 4000 deaths at the time, WHO declared the COVID-19 a pandemic. ${ }^{12}$ 
As the COVID-19 pandemic continued to unfold across the world, Jordan began detecting tens of cases, and decided to impose some of the strictest measures to combat an outbreak. On 14 March 2020, a travel ban was issued, and all borders in Jordan were blocked. Few days after, a strict nationwide lockdown was implemented, and residents were not allowed on the streets. ${ }^{3}$ Although lockdown ended in Wuhan, China on 8 April 2020 after 76 days of being implemented, ${ }^{4}$ Jordanians continued to live under some of the strictest measures of a lockdown for nearly 2 months until early May 2020. This included allowing some movement outside households for some days of the week while completely disallowing such movement on other days. Additionally, all parks, restaurants, cafes and any public open spaces were shut down indefinitely. Groceries were only available through delivery to households, and no cars could be driven on the streets in all regions of the country.

While a lockdown is considered a recommended measure in combating an outbreak, an extended lockdown may have severe effects on people's social lives, mobility and the overall economy. ${ }^{5}$ This includes influencing a set of health outcomes among the general population and could be an activator of stressors, such as anxiety, and several other symptoms of poor mental health. ${ }^{6-9}$ In the context of a prolonged strict lockdown measures, largescale social isolation is also induced among the population. In Jordan, a large proportion of the population is employed in working-class jobs, such as those in the industrial or agricultural sectors, which require manual labour and typically offer low pay. The lockdown has left many Jordanians unemployed and without the daily income they depend on, imposing significant financial stress. For instance, in a report demonstrating the impact of lockdown in Jordan on households across the country, three-quarters of respondents $(72.5 \%)$, among a sample of 12084 Jordanian adults, indicated having difficulties covering basic needs (rent, food, heating and medicine) due to the lockdown measures. ${ }^{3}$ Additionally, $63.3 \%$ indicated that they did not have a source of support in case they run out of food and basic amenities required for survival. $^{3}$

In general, previous research has consistently demonstrated that poor mental health is associated with multiple negative health outcomes, including poor sleep health. ${ }^{6-9}$ The Diagnostic and Statistical Manual of Mental Disorders, 5th Edition, widely used by psychiatrists across the globe, lists sleep disturbance as one of the possible symptoms required for the diagnosis of both major depressive disorder and generalised anxiety disorder. Negative mental health outcomes may be heightened in epidemics, such as during the COVID-19 pandemic. ${ }^{10}$ For instance, Wang et aldemonstrated that the COVID-19 pandemic and the restriction of freedom during lockdowns has been shown to have a significant psychological impact related to stress, depression and anxiety. ${ }^{11}$ Additionally, Brooks et al, reported that such psychosocial health outcomes (eg, stress, anger and fear) could be further increased during the state epidemic-induced lockdowns. ${ }^{12}$

Importantly, research has shown that sleep and the circadian system exert a strong regulatory influence on immune functions ${ }^{13}$ Sleep and the circadian system cooperate bidirectionally at the systemic and cellular level to organise immune functions in time and space via neuroendocrine and sympathetic effector mechanisms. ${ }^{14-17}$ The relationship between sleep health and the immune system is particularly of importance during times of epidemics. For example, Prather $e t$ al showed that among a sample of 164 healthy men and women, shorter sleep duration, measured behaviourally using actigraphy prior to viral exposure, was associated with increased susceptibility to the common cold. ${ }^{18}$ While there is a literature on the prevalence of mental health issues during the times of epidemics, ${ }^{19-24}$ as well as its relationship with sleep health among various populations, ${ }^{6-1025-27}$ few studies have investigated such an association during times of nationwide lockdowns, ${ }^{28-30}$ especially in the Middle East. Lockdown measures could be effective in controlling the transmission of infection, such measures could influence other health outcomes unrelated to their current outbreak, including sleep.

In this study, we sought to investigate the associations between mental health issues, namely anxiety and depressive symptoms, with poor sleep health among a sample of individuals residing in Jordan during a period of COVID-19 pandemic-induced lockdown. We hypothesised that anxiety and depressive symptoms will be associated with a higher risk of poor sleep health among a sample of Jordanians, after controlling for demographic covariates.

\section{METHODOLOGY}

\section{Data and study sample}

Facebook, a social media platform, was used to recruit a sample of individuals across Jordan during a period of nationwide lockdown put in place due to the COVID-19 pandemic outbreak in March 2020. Facebook was the platform of choice given its large spread among the Jordanian population, with approximately $83.21 \%$ of the population (9 903 802) being active users on Facebook in March 2020. ${ }^{31}$ An advertised post was placed on Facebook targeting Jordanians between the ages of 18 and 65 years who were in Jordan for the duration of the last 2 weeks of March 2020. The post was also placed by an independent researcher, and snowball sampling was also enabled by allowing the advertised post to be shared by individuals on Facebook. The survey was administered through Qualtrics, a platform for survey administration previously used in health research. ${ }^{32}$ The survey was available to participants in both English and Arabic languages. The survey was originally written in English, and then translated independently by three bilingual researchers to Arabic. The survey was then piloted for readability and clarity among both English and Arabic speakers. The post 
was advertised for a period of four consecutive days and included assessment of eligibility and a total of 30 items including sociodemographic and measures for various constructs. These included anxiety, depressive symptoms and sleep health. Measures were taken to avoid duplicate answers by using the 'prevent ballot box stuffing' feature on Qualtrics. Additionally, internal protocol addresses associated with responses were reviewed manually to ensure no duplicates were recorded. On average, the survey took $7 \mathrm{~min}$ for completion.

Overall, 3462 participants clicked on the link and were redirected to the survey, of which 2202 participants were eligible to participant. Twenty-two responses were excluded for being aged $<18$ years of age. Overall, a total of 1240 full responses were recorded, corresponding to a completion rate of $56.4 \%$ among those deemed eligible. Approximately two-thirds (67.4\%) of the respondents completed the survey in Arabic. Overall, the completion rate of those who clicked the linked to the survey was $36.5 \%$.

\section{Patient and public involvement}

No patient involved.

\section{Measures}

Anxiety

The General Anxiety Disorder 7-item (GAD-7) scale was used to measure anxiety among participants. The GAD-7 was developed to diagnose generalised anxiety disorders and has been validated in 2740 primary care patients. ${ }^{33}$ Seven items were included in the survey, and participants' anxiety was determined according to their scoring on the item. A 7-item questionnaire was used where each item asked participants how often, during the last 2 weeks, they were bothered by a given symptom. Response options were 'not at all', 'several days', 'more than half the days' and 'nearly every day' scored as $0,1,2$ and 3, respectively. Scores range from 0 to 21, with four categorisations of anxiety severity according to the score. Those who score between 0 and 4 were considered to have minimal anxiety, while those scoring between 5-9, 10-14 and 15-21 were noted to have mild, moderate and severe anxiety, respectively. Cronbach's alpha was 0.87 , indicating good internal consistency.

\section{Depressive symptoms}

Ten (10) items were adopted from the Center for Epidemiologic Studies Depression Scale. Those items were chosen to be consistent with cultural concerns regarding the topic of depression and to be appropriate when translated to the Arabic language. Response options were 'rarely or none of the time $(<1$ day $)$ ', 'some or a little of the time (1-2 days)', 'occasionally or a moderate amount of time (3-4 days)' and 'most or all of the time (5-7 days)', scored as $0,1,2$ and 3, respectively. Three items worded in the positive direction were reverse coded and all items were summed for a total score, with a possible range of $0-30$. During data analysis, the total scores were divided in quartiles: the first quartile (Q1: 0-3), the second quartile (Q2: 4-6), the third quartile (Q3: 7-11) and the fourth quartile (Q4: 12-27). Cronbach's alpha was 0.8145 , indicating good internal consistency.

\section{Sleep health}

Three items were adopted from the Pittsburgh Sleep Quality Index, a reliable and validated tool, ${ }^{34}$ and included in the survey to measure for sleep health outcomes: sleep quality, sleep duration and experiencing sleep problems. Sleep quality was measured using the item "During the past month, how would you rate your sleep quality overall?" ${ }^{35}$ Participants were asked to choose one of four options, including 'very good', 'fairly good', 'fairly bad' and 'very bad'. Consistent with previous research on sleep health, ${ }^{36-39}$ the four response options were dichotomised in statistical analysis good sleep quality (eg, includes 'very good' and 'fairly good') and poor sleep quality (eg, includes 'very bad' and 'fairly bad'). To measure sleep duration, we used the question asking, "During the past month, how many hours of actual sleep did you get each night? (This may be different from the number of hours you spent in bed.)" 35 Participants then reported their response in hours as integers with one decimal place allowed (eg, halves). Short sleep duration was defined as 7 hours or less. ${ }^{3640}$ Finally, sleep problems were assessed by asking the participants if they experienced any of three sleep-related problems in the past 2 weeks (eg, the period of lockdown). Three statements were presented, and participants were given a 'yes' or 'no' response options. These statements were (1) "I had trouble sleeping because I could not get to sleep within $30 \mathrm{~min}$ " (eg, problems falling asleep); (2) "I had trouble staying awake while driving, eating meals, or engaging in social activity" (eg, problems staying awake in the daytime also known as daytime sleepiness) and (3) "I took medicine (prescribed or 'over the counter') to help me sleep". ${ }^{37}$

\section{Physical activity}

Two items were adopted from the International Physical Activity Questionnaire (IPAQ) and included in this study. The IPAQ is a well-used instrument in health research, and has been shown to be reliable and valid across different populations. ${ }^{42-44}$ The two items used measured the number of days in which moderate-to-vigorous physical activity (MVPA) was performed, as well as the duration of time in minutes spent in a given day, respectively. The reported answers for both items were then multiplied to calculate the MVPA variable, defined as the duration (in minutes) spent in MVPA per one full week. The participants were then grouped based on whether they met the WHO's adults physical activity guidelines or not (eg, $150 \mathrm{~min}$ or more of MVPA per week). ${ }^{45} 46$

\section{Sociodemographic}

Four items were included to measure demographic variables among participants. Participants were asked to report their age (in years), gender (male or female), 
employment status (employed, self-employed, unemployed, student or retired) and the city they currently reside in within Jordan. Cities were grouped into the following regions: Northern region (Irbid, Jerash, Balqa'a, Zarqa, Ajloun and Mafraq), Central region (Amman, Madaba and Salt), Southern region (Tafila, Karak, Ma'an, Aqaba and Petra).

\section{Statistical analysis}

Descriptive statistics (frequencies, percentages) were calculated for sociodemographic characteristics, physical activity, anxiety and depression according to sleep health outcomes (eg, poor sleep quality, short sleep duration and experiencing sleep problems). Mann-Whitney $\mathrm{U}$ test or $\chi^{2}$ test was used to examine the differences in demographic characteristics, physical activity, anxiety and depression between those with and without each sleep health outcome.

The association between anxiety, depression and each poor sleep outcome was assessed using a modified Poisson regression model with robust error variance ${ }^{47}$ due to the high prevalence of the sleep health outcomes. ${ }^{48-53}$ The multivariable analysis adjusted for age, gender, employment status, region and physical activity. Adjusted prevalence ratios (aPRs) and 95\% CIs were estimated. Test for p-trend was calculated by treating the categories of anxiety and depression as continuous variables. Two-sided $\mathrm{p}<0.05$ was considered statistically significant. Data were analysed using Stata V.16.0 (StataCorp, College Station, Texas, USA).

\section{RESULTS}

Table 1 presents the participants' sociodemographic characteristics, anxiety and depressive symptoms according to sleep health outcomes. The mean age was 37.35 $(\mathrm{SD}=11.01)$, with over $60 \%$ of our sample aged $<40$ years. The median age of the sample was 35.0. Approximately half of the sample $(52.9 \%)$ were males. Additionally, more than three-quarters of the participants reported residing in the Central region $(78.2 \%)$, followed by the Northern region $(19.1 \%)$. Most of the participants were either employed (54.3\%) or self-employed (16.5\%), while $17.6 \%$ reported being unemployed or $4.6 \%$ were retired at the current time. Approximately a third of our sample $(31.5 \%)$ met the WHO's adults physical activity guidelines for MVPA of $150 \mathrm{~min}$ or more per week. In terms of anxiety, $53 \%$ of the participants reported symptoms for mild $(33.8 \%)$, moderate $(12.9 \%)$ or severe anxiety $(6.3 \%)$ during the period of national lockdown. Furthermore, approximately a quarter of our sample scored in each of the four quartiles for the depressive symptoms measures (eg, 26.9\% in the lowest quartile, $24.8 \%$ in the second quartile, $26.8 \%$ in the third quartile and $21.5 \%$ in the highest quartile). Finally, and when measuring for sleep health outcomes, $17.9 \%$ of the participants reported poor sleep quality, $46.8 \%$ reported short sleep duration $(\leq 7$ hours of sleep on average for every night over the past week), while $63.7 \%$ reported experiencing at least one sleep problem over the past week.

Multivariable regressions with 95\% CIs were calculated to investigate the relationship between anxiety, depressive symptoms and sleep health outcomes. The results, after adjusting for sociodemographic covariates, are presented in table 2. Anxiety was associated with poor sleep health outcomes. Results demonstrated a dose-response relationship, as the aPRs for poor sleep health increased with increasing levels of both anxiety and depressive symptoms. Compared with those who reported minimal anxiety, participants reporting mild anxiety were more likely to experience poor sleep quality $(\mathrm{aPR}=3.01 ; 95 \% \mathrm{CI}=2.07$ to 4.35 ), short sleep duration ( $\mathrm{aPR}=1.35 ; 95 \% \mathrm{CI}=1.17$ to 1.56) and at least one problem sleep problem $(\mathrm{aPR}=1.41$; $95 \% \mathrm{CI}=1.27$ to 1.56 ). Similarly, those reporting moderate anxiety were more likely to experience poor sleep quality (aPR $=5.78 ; 95 \% \mathrm{CI}=3.97$ to 8.43 ), short sleep duration $(\mathrm{aPR}=1.73 ; 95 \% \mathrm{CI}=1.47$ to 2.04$)$ and at least one problem sleep problem $(\mathrm{aPR}=1.56 ; 95 \% \mathrm{CI}=1.39$ to 1.75$)$ compared with those reporting minimal anxiety. Similarly, and corresponding to the dose-response relationship between anxiety and sleep health outcomes, those reporting severe anxiety were the most likely to experience poor sleep quality $(\mathrm{aPR}=8.954 ; 95 \% \mathrm{CI}=6.12$ to 13.08), short sleep duration ( $\mathrm{aPR}=2.23$; $95 \% \mathrm{CI}=1.91$ to $2.61)$ and at least one problem sleep problem $(\mathrm{aPR}=1.73$; $95 \% \mathrm{CI}=1.54$ to 1.95$)$. P-trend $<0.001$ for all sleep outcomes.

Depressive symptoms were also associated with poor sleep health outcomes. This association also demonstrated dose-response relationship. As compared with scoring in the first quartile, scoring in the second, third and fourth quartile was significantly associated with poor sleep quality ( $\mathrm{aPRs}=2.19,5.27$ and 11.82 , respectively), short sleep duration (aPRs=1.21, 1.41 and 1.87, respectively) and experiencing at least one sleep problem (aPRs=1.40, 1.62 and 1.90, respectively).

\section{DISCUSSION}

This study is the first to empirically explore both mental health and sleep health measures during nationwide lockdown in response to the COVID-19 pandemic. It is also the first study to evaluate associations between mental health and sleep health in Jordan, and one of few studies to address the relationship between these measures among a severely understudied population; Middle Eastern adults. To the best of our knowledge, there is only one other study that has assessed sleep health in Jordan, which reported that poor sleep health, including a high risk of obstructive sleep apnoea, snoring and daytime sleepiness, was common among Jordanians attending primary care clinics. ${ }^{54}$

In our study, the majority of participants reported having experienced mild (33.8\%), moderate $(12.9 \%)$ or severe $(6.3 \%)$ levels of anxiety during lockdown, and nearly half of respondents reported depressive symptoms 


$$
\text { . }
$$$$
\text { 을. }
$$ 


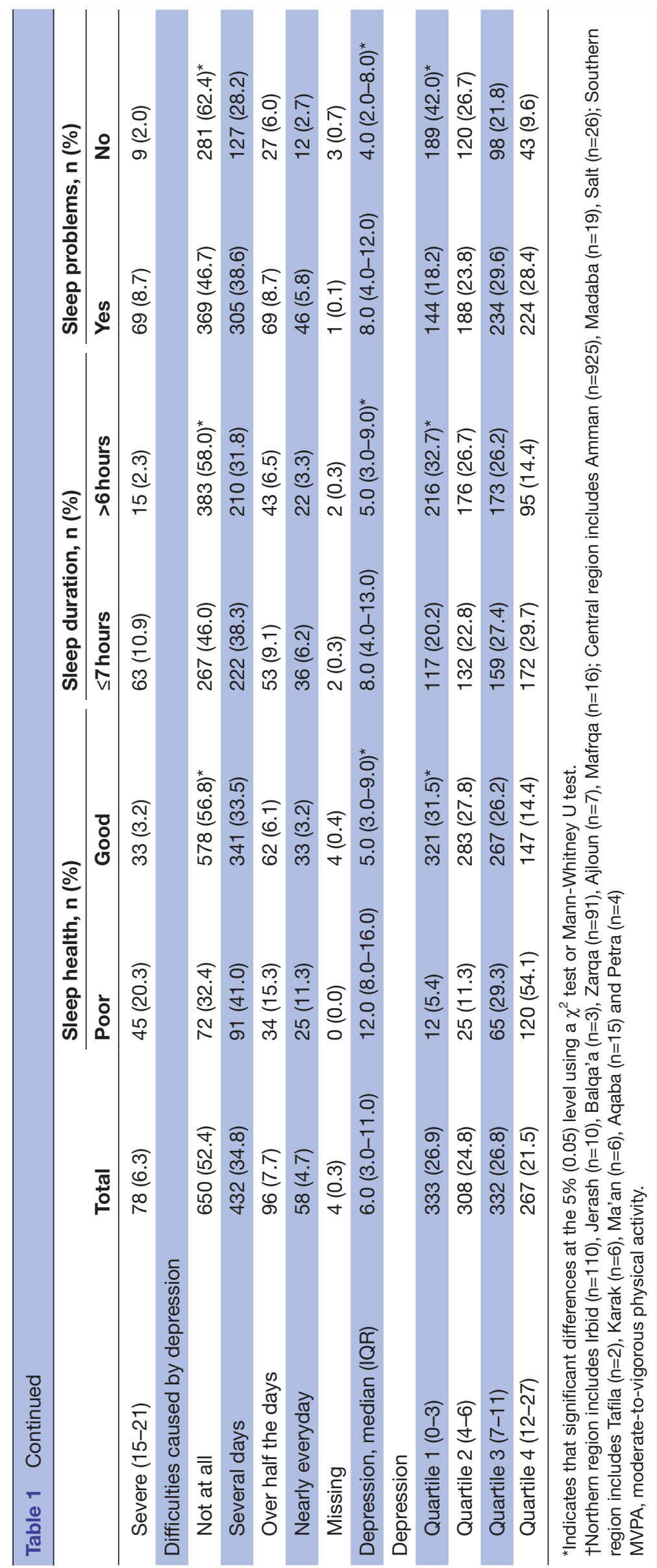


Table 2 Multivariable association (aPRs)† between anxiety, depression and poor sleep health

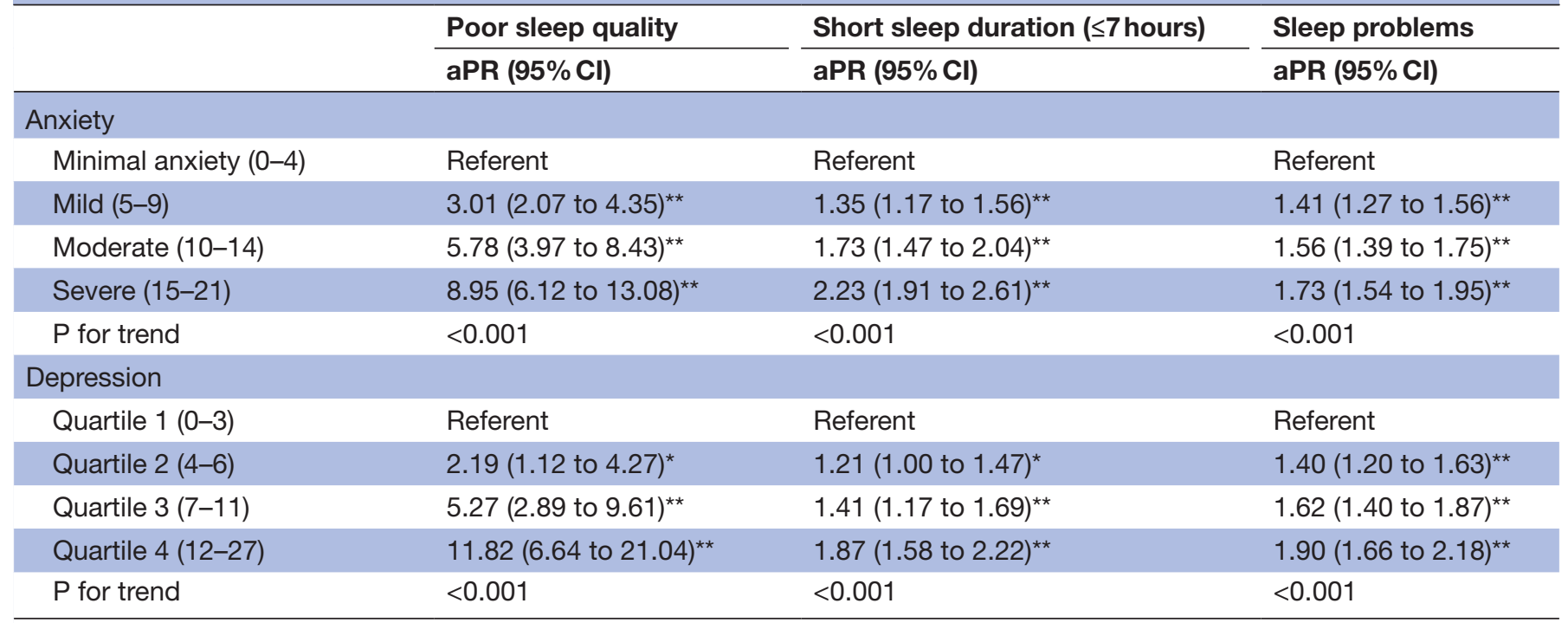

${ }^{*} \mathrm{P}<0.05 ;{ }^{* *} \mathrm{p}<0.001$

†Adjusted for age, gender, region, employment and physical activity aPR, adjusted prevalence ratio.

during lockdown. Similarly, over $60 \%$ of participants reported having experienced at least one sleep problem in the last week, and nearly half reported having had short sleep duration. In addition, we found that increased levels of reported anxiety or depression were both significantly associated with an increase in poor sleep health outcomes among Jordanians during lockdown in response to the COVID-19 pandemic.

Our findings that increased levels of self-reported anxiety or depression were significantly associated with an increase in poor sleep health outcomes can potentially be explained by the relationship between mental health and sleep health. The pathway by which depression alters sleep is thought to be through the loss of deep, slow-wave sleep and an increase in nocturnal arousal. The combination of these two effects contributes to a decrease in non-REM sleep, which may subsequently result in reduced REM sleep latency. ${ }^{55}$ Several studies have shown the association between depression and the disruption of REM sleep. ${ }^{56} 57$ Furthermore, the cause of depression is most commonly attributed to the dysregulation of monoamines, such as serotonin, dopamine, norepinephrine and dopamine. An individual's transition to REM sleep is typically accompanied by a rapid decrease in these monoamines, ${ }^{58}$ and such dysregulation may be associated with the disruption of REM sleep and the subsequent onset of depressive symptoms . Research has also demonstrated that depression is correlated with increased inflammatory markers and that depressive symptoms can be reduced by decreasing that inflammation. ${ }^{59}$ As such, sleep loss has been associated with the increased production of inflammatory markers, such as C reactive protein and interleukins. ${ }^{60}$ Although the exact mechanism is not fully understood, there appears to be a relationship between sleep disturbance, inflammation and depression. ${ }^{5961}$ Another potential pathway by which depression and sleep disturbances interact is through alternations in one's circadian rhythm. An individual's circadian rhythm is crucial to maintaining the sleep-wake cycle, and it has been observed that depression disrupts circadian rhythm. ${ }^{62}$

Overall, our findings suggest the possible impact that economic, social or health-related circumstances caused by nationwide lockdown can have in exacerbating anxiety, depressive symptoms and poor sleep health among respondents in Jordan. Particular to the context of lockdown in response to the COVID-19 pandemic, the Jordanian government did not provide economic support, financial incentives (eg, stimulus) or loan forgiveness schemes to support individuals who may have lost significant portions of their income or faced additional financial obligations as a result of lockdown and their inability to engage with their given occupations consistently. ${ }^{3}$ As a result, these economic circumstances may have contributed to increased anxiety, reported depressive symptoms and poor sleep health. While it is expected that these economic circumstances would affect adult men in particular because they are typically considered financial providers for their families in the context of Jordanian culture $^{63}$ reported rates of anxiety, depressive symptoms and poor sleep health were comparable between male and female respondents. This is the case despite that within Jordan, a larger proportion of men work outside the home than women. That being said, because childcare, elderly care and housework are typically women's responsibilities in this context, the lockdown restrictions would be expected to disproportionately affect women's economic resilience, mental well-being and sleep health, especially given rising levels of domestic violence and reduced access to sexual and reproductive health services 
during lockdown. ${ }^{64}{ }^{65}$ It should be noted that studies have shown that men tend to have a higher prevalence of sleep disorders such as obstructive sleep apnoea, other studies have found that women are more likely than men to report an increased burden of depressive symptoms as a result of poor sleep health. ${ }^{66}$

Other factors that have been found to affect sleep health and mental health outcomes that are also expected to be relevant or exacerbated during lockdown situations include tobacco or khat use, atypical work schedules and violent or poor familial environments in households. ${ }^{67-71}$ The increased recreational screen time during lockdown spent watching television, using a computer or using smartphones and the reality of isolation with limited social contact would also be expected to contribute to poor mental health outcomes, including anxiety and depressive symptoms, found in our study. ${ }^{71-73}$ In addition, unemployment has been noted to be associated with diminished mental health and is negatively correlated with sleep health measures. ${ }^{74}$ Approximately $25 \%$ of unemployed or retired respondents in our study reported having experienced at least one sleep problem in the last week and short sleep duration. These risk factors are expected to work in tandem and are expected to expose underlying vulnerabilities that also contribute to poor mental health and sleep health. ${ }^{74-76}$

When evaluating the potential consequences of lockdown during a pandemic such as COVID-19, it is critical to consider how lockdown may predispose individuals to risk factors that impact mental health and sleep health. Poor sleep habits, anxiety and depression are all recognised as affecting overall health and contributing to a number of risk factors for cardiovascular disease, obesity, hypertension, hyperlipidaemia and diabetes, among other chronic conditions that are increasingly prevalent in Jordan. ${ }^{77}$ Similarly, poor mental health and sleep health are also found to be associated with excessive alcohol use and substance use ${ }^{678}$ Given that poor sleep habits, anxiety and depression are also associated with decreased immune responsiveness, it is especially important to consider how the impacts of nationwide lockdown on mental health and sleep health may make individuals particularly vulnerable to SARS-CoV-2, the causative agent of COVID-19, or other highly infectious pathogens. ${ }^{79}$

The study findings, the lack of interventions in place to support the population during strict lockdowns (eg, financial incentives, mental health resources), and the absence of discourse to address poor sleep health or mental health as a result of nationwide lockdown highlight a gap in policymaking and planning during health emergencies and disease outbreaks in Jordan and likely in other countries. While the impact of lockdown on mental health and sleep health is not expected to be unique to Jordan, future research should focus on further elucidating the determinants of mental health and poor sleep health during lockdown and exploring the potential interactive impacts of specific risk factors that are exacerbated during lockdown on mental health and sleep health and their subsequent effects on susceptibility to disease. Additionally, the mechanism(s) in which such association occurs should be investigated. Future attention should also focus on articulating policies and designing interventions to address identified determinants and risk factors and to uphold an international standard of individuals' right to health, ${ }^{80}$ especially for vulnerable and disadvantaged populations.

\section{Limitations}

This study's methodology is subject to several limitations. First, the sample population only includes individuals using one social media platform, which introduces self-selection and volunteer bias and limits the generalisability of this study. Second, the information collected using the online survey includes exclusively self-reported data. As a result, responses, especially those measuring sleep health and mental health, can be implicated with decreasing the reliability and/or the validity of the study findings. Third, because our analysis did not adjust for additional confounding factors, such as substance use, education and marital status, residual confounding may have been introduced. However, we note that we used a limited survey to increase the survey completion rate. Finally, given that this study uses a cross-sectional survey design, it cannot be used to analyse mental health and sleep health measures over an extended period of time. Consequently, causal inference is limited.

\section{CONCLUSIONS}

This study provides evidence of reported anxiety and depressive symptoms and their association with poor sleep health outcomes during lockdown in Jordan in the face of the COVID-19 pandemic. Our findings draw attention to the potential negative consequences of lockdown and their impact on sleep health, emphasising the need for public health professionals and governments to monitor health profiles during lockdown and to create interventions aimed at improving sleep health and wellbeing. More attention is required to investigate the impacts of lockdown in exacerbating risk factors for poor mental health and sleep health, and future interventions and policies should be tailored towards addressing identified risk factors and improving health outcomes in the context of a nationwide lockdown.

\section{Author affiliations}

${ }^{1}$ Department of Primary Care and Public Health, Cambridge University, Cambridge, UK

${ }^{2}$ Department of Epidemiology, Columbia University Mailman School of Public Health, New York City, New York, USA

${ }^{3}$ Institute of Public Health, University of Cambridge, Cambridge, UK

${ }^{4}$ Department of Cell and Molecular Biology, Tulane University School of Science and Engineering, New Orleans, Louisiana, USA

${ }^{5}$ Presbyterian Hospital, New York City, New York, USA

${ }^{6}$ ICAP at Columbia University, New York City, New York, USA

Twitter Yazan A Al-Ajlouni @AjlouniYazan 
Contributors YAA-A contributed to the design of this research work. GS was involved in data collection for this project. YAA-A and SHP was involved in data analysis and interpretation. YAA-A, SHP, JA, AB and DTD were involved in drafting the article. WME-S and DTD provided critical revision of the article. YAA-A, SHP, JA, $G S, A B, W M E-S$ and DTD approved the final version of the manuscript.

Funding The authors have not declared a specific grant for this research from any funding agency in the public, commercial or not-for-profit sectors.

Competing interests None declared.

Patient consent for publication Not required.

Ethics approval All protocols and procedures were approved by the University of Jordan Hospital Institutional Review Board prior to data collection.

Provenance and peer review Not commissioned; externally peer reviewed.

Data availability statement Data are available on reasonable request.

Open access This is an open access article distributed in accordance with the Creative Commons Attribution Non Commercial (CC BY-NC 4.0) license, which permits others to distribute, remix, adapt, build upon this work non-commercially, and license their derivative works on different terms, provided the original work is properly cited, appropriate credit is given, any changes made indicated, and the use is non-commercial. See: http://creativecommons.org/licenses/by-nc/4.0/.

ORCID iDs

Yazan A Al-Ajlouni http://orcid.org/0000-0002-5527-7520

Su Hyun Park http://orcid.org/0000-0002-5527-7520

Jude Alawa http://orcid.org/0000-0003-3600-4356

\section{REFERENCES}

1 Caso V, Federico A. No lockdown for neurological diseases during COVID19 pandemic infection. . Springer, 2020: 41. 999-1001.

2 WHO Director-General's opening remarks at the media briefing on COVID-19 - 11 2020;2020.

3 UNDP Jordan. Impact of COVID-19 on households in Jordan, 2020.

4 Lee I-K, Wang C-C, Lin M-C, et al. Effective strategies to prevent coronavirus disease-2019 (COVID-19) outbreak in hospital. J Hosp Infect 2020;105:102-3.

5 Shrestha AM, Shrestha UB, Sharma R, et al. Lockdown caused by COVID-19 pandemic reduces air pollution in cities worldwide, 2020.

6 Kenney SR, Lac A, LaBrie JW, et al. Mental health, sleep quality, drinking motives, and alcohol-related consequences: a path-analytic model. J Stud Alcohol Drugs 2013;74:841-51.

7 Tsuno N, Besset A, Ritchie K. Sleep and depression. J Clin Psychiatry 2005;66:1254-69.

8 Augner $\mathrm{C}$. Associations of subjective sleep quality with depression score, anxiety, physical symptoms and sleep onset latency in young students. Cent Eur J Public Health 2011;19:115-7.

9 Duncan DT, Goedel WC, Mayer KH, et al. Poor sleep health and its association with mental health, substance use, and condomless anal intercourse among gay, bisexual, and other men who have sex with men. Sleep Health 2016;2:316-21.

10 Hao F, Tan W, Jiang L, et al. Do psychiatric patients experience more psychiatric symptoms during COVID-19 pandemic and lockdown? A case-control study with service and research implications for immunopsychiatry. Brain Behav Immun 2020;87:100-6.

11 Wang C, Pan R, Wan X, et al. Immediate psychological responses and associated factors during the initial stage of the 2019 coronavirus disease (COVID-19) epidemic among the general population in China. Int J Environ Res Public Health 2020;17:1729.

12 Brooks SK, Webster RK, Smith LE, et al. The psychological impact of quarantine and how to reduce it: rapid review of the evidence. The Lancet 2020;395:912-20.

13 Besedovsky L, Lange T, Born J. Sleep and immune function. Pflugers Arch - Eur J Physiol 2012;463:121-37.

14 Marshall L, Born J. Brain-Immune interactions in sleep. Int Rev Neurobiol 2002:52:93-131.

15 Arjona A, Sarkar DK. Are circadian rhythms the code of hypothalamic-immune communication? Insights from natural killer cells. Neurochem Res 2008;33:708-18.

16 Coogan AN, Wyse CA. Neuroimmunology of the circadian clock. Brain Res 2008;1232:104-12.

17 Imeri L, Opp MR, How OMR. How (and why) the immune system makes us sleep. Nat Rev Neurosci 2009;10:199-210.

18 Prather AA, Janicki-Deverts $\mathrm{D}$, Hall $\mathrm{MH}$, et al. Behaviorally assessed sleep and susceptibility to the common cold. Sleep 2015;38:1353-9.
19 Alexander MJ, Trauma MK. Addiction and recovery: Addressing public health epidemics among women with severe mental illness, 1998.

20 Chatterjee K, Chauhan VS. Epidemics, quarantine and mental health. Med J Armed Forces India 2020;76:125-7.

21 Vigo D, Patten S, Pajer K, et al. Mental health of communities during the COVID-19 pandemic. Los Angeles, CA: SAGE Publications Sage CA, 2020: 65. 681-7.

22 Shi L, Lu Z-A, Que J-Y, et al. Prevalence of and risk factors associated with mental health symptoms among the general population in China during the coronavirus disease 2019 pandemic. JAMA Netw Open 2020;3:e2014053.

23 Pappa S, Ntella V, Giannakas T, et al. Prevalence of depression, anxiety, and insomnia among healthcare workers during the COVID-19 pandemic: a systematic review and meta-analysis. Brain Behav Immun 2020;88:901-7.

24 Ćosić K, Popović S, Šarlija M, et al. Impact of human disasters and Covid-19 pandemic on mental health: potential of digital psychiatry. Psychiatr Danub 2020;32:25-31.

25 Zandifar A, Badrfam R. Iranian mental health during the COVID-19 epidemic. Asian J Psychiatr 2020;51:101990.

26 Cao W, Fang Z, Hou G, et al. The psychological impact of the COVID-19 epidemic on college students in China. Psychiatry Res 2020;287:112934.

27 Roy D, Tripathy S, Kar SK, et al. Study of knowledge, attitude, anxiety \& perceived mental healthcare need in Indian population during COVID-19 pandemic. Asian J Psychiatr 2020;51:102083.

28 Gualano MR, Lo Moro G, Voglino G, et al. Effects of Covid-19 lockdown on mental health and sleep disturbances in Italy. Int $J$ Environ Res Public Health 2020;17:4779.

29 Huang Y, Zhao N. Generalized anxiety disorder, depressive symptoms and sleep quality during COVID-19 outbreak in China: a web-based cross-sectional survey. Psychiatry Res 2020;288:112954.

30 Zhang C, Yang L, Liu S, et al. Survey of insomnia and related social psychological factors among medical staff involved in the 2019 novel coronavirus disease outbreak. Front Psychiatry 2020;11:306.

31 Stats Counter. Social Media Stats in Jordan - March 2020, 2020.

32 Qualtrics L. Qualtrics [software]. Utah, USA: Qualtrics, 2014.

33 Spitzer RL, Kroenke K, Williams JBW, et al. A brief measure for assessing generalized anxiety disorder: the GAD-7. Arch Intern Med 2006;166:1092-7.

34 Mollayeva T, Thurairajah P, Burton K, et al. The Pittsburgh sleep quality index as a screening tool for sleep dysfunction in clinical and non-clinical samples: a systematic review and meta-analysis. Sleep Med Rev 2016;25:52-73.

35 Buysse DJ, Reynolds CF, Monk TH, et al. The Pittsburgh sleep quality index: a new instrument for psychiatric practice and research Psychiatry Res 1989;28:193-213.

36 Duncan DT, Hyun Park S, Al-Ajlouni YA, et al. Association of financial hardship with poor sleep health outcomes among men who have sex with men. SSM Popul Health 2017;3:594-9.

37 Duncan DT, Park SH, Goedel WC, et al. Perceived neighborhood safety is associated with poor sleep health among gay, bisexual, and other men who have sex with men in Paris, France. $J$ Urban Health 2017;94:399-407.

38 Millar BM, Parsons JT, Redline S, et al. What's sleep got to do with it?: Sleep health and sexual risk-taking among men who have sex with men. AIDS Behav 2019;23:572-9.

39 Ruff RR, Ng J, Jean-Louis G, et al. Neighborhood stigma and sleep: findings from a pilot study of low-income housing residents in New York City. Behav Med 2018;44:48-53.

40 Consensus Conference Panel, Watson NF, Badr MS, et al. Joint consensus statement of the American Academy of sleep medicine and sleep research Society on the recommended amount of sleep for a healthy adult: methodology and discussion. Sleep 2015;38:1161-83.

41 Mountcastle HD, Park SH, Al-Ajlouni YA, et al. Stress levels are associated with poor sleep health among sexual minority men in Paris, France. Sleep Health 2018;4:436-41.

42 Craig CL, Marshall AL, Sjöström M, et al. International physical activity questionnaire: 12 -country reliability and validity. Med Sci Sports Exerc 2003;35:1381-95.

43 Hagströmer M, Oja P, Sjöström M. The International physical activity questionnaire (IPAQ): a study of concurrent and construct validity. Public Health Nutr 2006;9:755-62.

44 Lee PH, Macfarlane DJ, Lam TH, et al. Validity of the International physical activity questionnaire short form (IPAQ-SF): a systematic review. Int J Behav Nutr Phys Act 2011;8:115.

45 Tucker JM, Welk GJ, Beyler NK. Physical activity in U.S.: adults compliance with the physical activity guidelines for Americans. Am J Prev Med 2011;40:454-61. 
46 World Health Organization. Recommended levels of physical activity for adults aged 18 - 64 years. Physical Activity and Adults Web site.

47 Zou G. A modified poisson regression approach to prospective studies with binary data. Am J Epidemiol 2004;159:702-6.

48 Behrens T, Taeger D, Wellmann J, et al. Different methods to calculate effect estimates in cross-sectional studies. A comparison between prevalence odds ratio and prevalence ratio. Methods Inf Med 2004;43:505-9.

49 McNutt L-A, Wu C, Xue X, et al. Estimating the relative risk in cohort studies and clinical trials of common outcomes. Am J Epidemiol 2003;157:940-3.

50 Thompson ML, Myers JE, Kriebel D. Prevalence odds ratio or prevalence ratio in the analysis of cross sectional data: what is to be done? Occup Environ Med 1998;55:272-7.

51 Spiegelman D, Hertzmark E. Easy SAS calculations for risk or prevalence ratios and differences. Am J Epidemiol 2005;162:199-200.

52 skove T, Deddens J, Petersen MR, et al. Prevalence proportion ratios: estimation and hypothesis testing. Int J Epidemiol 1998;27:91-5.

53 Halkin A, Reichman J, Schwaber M. Likelihood ratios: getting diagnostic testing into perspective. QJM 1998;91:247-58.

54 Khassawneh B, Ghazzawi M, Khader Y, et al. Symptoms and risk of obstructive sleep apnea in primary care patients in Jordan. Sleep and Breathing 2009;13:227-32.

55 Sadock BJ, Sadock VA. Kaplan \& Sadock's concise textbook of clinical psychiatry. Lippincott Williams \& Wilkins, 2008.

56 Lustberg L, Reynolds CF. Depression and insomnia: questions of cause and effect. Sleep Med Rev 2000;4:253-62.

57 Adrien J. Neurobiological bases for the relation between sleep and depression. Sleep Med Rev 2002;6:341-51.

58 Pace-Schott EF, Hobson JA. The neurobiology of sleep: genetics, cellular physiology and subcortical networks. Nat Rev Neurosci 2002;3:591-605.

59 Raison CL, Rutherford RE, Woolwine BJ, et al. A randomized controlled trial of the tumor necrosis factor antagonist infliximab for treatment-resistant depression: the role of baseline inflammatory biomarkers. JAMA Psychiatry 2013;70:31-41.

60 Irwin MR, Wang M, Campomayor CO. Sleep deprivation and activation of morning levels of cellular and genomic markers of inflammation. Arch Intern Med 2006;166:1756-62.

61 Fang H, Tu S, Sheng J, et al. Depression in sleep disturbance: a review on a bidirectional relationship, mechanisms and treatment. J Cell Mol Med 2019;23:2324-32.

62 JZ L, Bunney BG, Meng F, et al. Circadian patterns of gene expression in the human brain and disruption in major depressive disorder. Proceedings of the National Academy of Sciences 2013;110:9950-5.

63 Miles R. Employment and unemployment in Jordan: the importance of the gender system. World Dev 2002;30:413-27.
64 The Lancet. The gendered dimensions of COVID-19. The Lancet 2020;395:1168.

65 Park EM, Meltzer-Brody S, Stickgold R. Poor sleep maintenance and subjective sleep quality are associated with postpartum maternal depression symptom severity. Arch Womens Ment Health 2013;16:539-47.

66 Boccabella A, Malouf J. How do sleep-related health problems affect functional status according to sex? J Clin Sleep Med 2017;13:685-92.

67 Nakajima M, Dokam A, Kasim AN, et al. Habitual khat and concurrent khat and tobacco use are associated with subjective sleep quality. Prev Chronic Dis 2014;11.

68 Lin P-C, Chen C-H, Pan S-M, et al. Atypical work schedules are associated with poor sleep quality and mental health in Taiwan female nurses. Int Arch Occup Environ Health 2012:85:877-84.

69 Sankri-Tarbichi AG. Obstructive sleep apnea-hypopnea syndrome: etiology and diagnosis. Avicenna J Med 2012;2:3.

70 Montgomery E, Foldspang A. Traumatic experience and sleep disturbance in refugee children from the middle East. Eur J Public Health 2001;11:18-22.

71 Lubans D, Richards J, Hillman C, et al. Physical activity for cognitive and mental health in youth: a systematic review of mechanisms. Pediatrics 2016;138:e20161642.

72 Babic MJ, Smith JJ, Morgan PJ, et al. Longitudinal associations between changes in screen-time and mental health outcomes in adolescents. Ment Health Phys Act 2017;12:124-31.

73 Wu X, Tao S, Zhang Y, et al. Low physical activity and high screen time can increase the risks of mental health problems and poor sleep quality among Chinese college students. PLoS One 2015;10:e0119607.

74 Strandh M, Winefield A, Nilsson K, et al. Unemployment and mental health scarring during the life course. Eur J Public Health 2014;24:440-5.

75 Björklund A, Eriksson T. Unemployment and mental health: evidence from research in the Nordic countries. Scand J Soc Welf 1998;7:219-35.

76 Antillón M, Lauderdale DS, Mullahy J. Sleep behavior and unemployment conditions. Econ Hum Biol 2014;14:22-32.

77 Vats MG, Mahboub BH, Al Hariri H, et al. Obesity and sleeprelated breathing disorders in middle East and UAE. Can Respir J 2016;2016:1-5.

78 Johnson EO, Breslau N. Sleep problems and substance use in adolescence. Drug Alcohol Depend 2001;64:1-7.

79 Irwin MR. Why sleep is important for health: a psychoneuroimmunology perspective. Annu Rev Psychol 2015;66:143-72.

80 Pūras D, de Mesquita JB, Cabal L, et al. The right to health must guide responses to COVID-19. Lancet 2020;395:1888-90. 